\title{
Incorporation of potent and selective cytochrome P450 1B1 inhibitors into Ru(II) scaffolds provides unprecedented control over enzyme activity with light
}

\section{Dmytro Havrylyuk}

University of Kentucky

Austin Hachey

Massachusetts General Hospital

Alexander Fenton

University of Kentucky

David Heidary

University of Kentucky

Edith Glazer ( $\square$ ec.glazer@uky.edu )

University of Kentucky

\section{Article}

Keywords: CYP1B1, chemotherapy efficacy, prodrugs

Posted Date: October 15th, 2021

DOI: https://doi.org/10.21203/rs.3.rs-927533/v1

License: (c) (i) This work is licensed under a Creative Commons Attribution 4.0 International License.

Read Full License 


\section{Abstract}

The cytochrome P450 family of enzymes (CYPs) are important targets for medicinal chemistry. Recently, CYP1B1 has emerged as a key player in chemotherapy resistance in the treatment of cancer. This enzyme is overexpressed in a variety of tumors, and is correlated with poor treatment outcomes; thus, it is desirable to develop CYP1B1 inhibitors to restore chemotherapy efficacy. However, possible off-target effects, such as inhibition of liver CYPs responsible for first pass metabolism, make selective inhibition a high priority to avoid possible drug-drug interactions and toxicity. Here we describe the creation of lighttriggered CYP1B1 inhibitors as "prodrugs", and achieve $>6,000$-fold improvement in potency upon activation with low energy $(660 \mathrm{~nm})$ light. The novel systems provide a selectivity index of 4,000100,000 over other off-target CYPs. One key to the design was the development of novel, coordinating CYP1B1 inhibitors which suppress enzyme activity at pM concentrations in live cells. The coordinating group enforces inhibitor orientation in the active site by anchoring to the iron. The second essential component was the biologically compatible Ru(II) scaffold that cages the inhibitors before photochemical release. These $\mathrm{Ru}(\mathrm{II})$ photocages are anticipated to provide similar selectivity and control for any coordinating CYP inhibitors.

\section{Introduction}

Cytochrome P450s (CYPs) are a superfamily of enzymes involved in a variety of processes essential for health, including metabolism of xenobiotics and biosynthesis of important signaling molecules such as hormones. ${ }^{1}$ However, imbalances in their activity, and/or cellular responses to their metabolic products, are important drivers in a number of disease states. A paradigm for the disease-associated activity of CYPs is CYP19A1, also known as aromatase, which generates estrogen, and thus provides the fuel for estrogen-driven cancers. When the aromatase inhibitors anastrozole, letrozole, and exemestane were introduced into clinical use, it resulted in profound extension in survival for estrogen receptor positive (ER+) breast cancer patients. ${ }^{2}$

Several moderately selective CYP inhibitors are accepted drugs, but a major medical concern is always the possibility of off-target effects. ${ }^{3}$ For example, systemic use of azole antifungal agents that target fungal CYP51 can result in disruption of essential steroid metabolizing CYPs and others involved in drug metabolism. ${ }^{4,5}$ Humans express 57 active CYPs, subclassified into 18 families, and all share the same P450 fold and active site. In addition to the inherent structural and functional diversity displayed by the wild type enzymes, polymorphisms are common, with more than 1,000 single nucleotide variants known for CYP2D6 alone. ${ }^{6}$ Thus, the vast variation of CYPs makes it challenging to predict the impact of seemingly selective CYP inhibitors on individual patients.

One option to increase control and selectivity is to develop CYP inhibitors as photolabile prodrugs to allow for release in targeted tissues, using radiation. This approach requires that the protected form be unable to inhibit the enzyme, which has proven to be surprisingly difficult. Smaller organic protecting groups are inadequate, as the linkage that provides for the photoreactivity is also subject to cleavage by 
CYPs. For example, coumarins, which are excellent photocages, are substrates of CYPs. ${ }^{78}$ Efforts from both our ${ }^{9}$ and other groups ${ }^{10,11}$ have focused on very large metal complexes as protecting groups. However, even "protected" CYP inhibitors have demonstrated significant activity. This is particularly surprising when the moiety thought to bind to the heme in the deeply buried active site is coordinated to the metal carrier, thus preventing the inhibitor's preferred binding orientation. ${ }^{10-13}$ Moreover, the addition of the metal center and its associated ligands takes up $400-515 \AA^{3}$ of space ${ }^{14}$ within the CYP active site or access channel. Some CYPs are able to accommodate such large groups, as was demonstrated with the various crystal structures of P450s containing large substrate- or inhibitor-bound moities, ${ }^{15-18}$ but it requires extraordinary flexibility of the enzyme, given the buried and hydrophobic nature of the active site. As a result of these various features, photocontrol of CYP inhibitors has been an unrealized goal.

We have an interest in the cancer-associated CYP named CYP1B1. This extrahepatic enzyme is overexpressed in a variety of tumors and converts estrogen to DNA mutagens. ${ }^{19}$ Moreover, CYP1B1 overexpression and point mutations result in resistance to many chemotherapeutics, such as cisplatin, ${ }^{20}$ daunorubicin, ${ }^{21}$ and taxanes. ${ }^{22-25}$ Given the lack of connection between the mechanisms of action of these essential drugs, which form the foundation of the vast majority of chemotherapy regimens, CYP1B1-mediated resistance may be negatively impacting treatment outcomes for most cancer patients who receive chemotherapy. Here, we report the successful development of small molecule inhibitors of CYP1B1 based on a coordination-mediated prodrug strategy.

\section{Results}

Synthesis of coordinating CYP1B1 inhibitors. While inhibitors containing a coordinating moiety to bind the iron heme are available for various other CYPs, ${ }^{26}$ CYP1B1 inhibitors containing coordinating groups that may be used in photocaging have not yet been developed. Moreover, addition of coordinating groups to any CYP inhibitor is a risky strategy, as off-target CYP inhibition can occur simply due to the presence of the iron-binding entity. Accordingly, we chose to base our design on a relatively simple and small known CYP1B1 inhibitor, tetramethoxystilbene (TMS; 1, Chart 1), as we could generate multiple derivatives, if needed, to establish selectivity. We predicted that only one of the methoxy-containing rings was required to engage active site residues, which allowed for incorporation of Lewis bases at the opposite end of the molecule to form a dative bond to the iron. The coordinating group ensured proper orientation within the active site by anchoring the inhibitor in place, and the ring system distal to the heme could be the site of potential systematic modification.

For simplicity and maximal generalizability to other drug molecules, nitrogen heterocycles where chosen as the Lewis donors. It was expected that the diazine ring in compound $\mathbf{2}$ would provide for superior photochemistry in the Ru(II) complex, ${ }^{27}$ while the pyridyl ring in $\mathbf{3}$ would produce a more potent CYP inhibitor, due to its greater basicity, which increases the coordinative bond strength to the iron. Both compounds $\mathbf{2}$ and $\mathbf{3}$ were synthesized in a single step using a modified Knoevenagel reaction. 
Validation of CYP1B1 coordinating inhibitors. To rigorously assess the activity of inhibitors under biologically relevant conditions, an assay was developed for screening of CYP1B1 activity in live cells. The use of an in cell assay, rather than the common CYP assays that utilize microsomes, was motivated by multiple factors. First, CYPs are known to engage various hydrophobic entities, such as detergents, and investigations with purified proteins are likely to generate false positives, with inhibition due to nonselective interactions that would not occur in the cellular setting. In addition, cellular uptake is a key feature for biological utility of new molecular agents. Finally, cell-based assays provide information on non-specific interactions that could be damaging to cell health. Given the need for CYP inhibitors to be selective and non-toxic, cell-based screens provide immediate feedback on these essential characteristics.

Table 1. Inhibitory potencies and selectivity indices (SI) of stilbene derivatives.

\begin{tabular}{|c|c|c|c|c|c|c|c|c|c|c|c|}
\hline$\overline{\mathrm{Cmpd}}$ & $\begin{array}{r}\text { IC50 C) } \\
(\mathrm{ml}\end{array}$ & $\begin{array}{l}\text { YP1B1 } \\
\text { M) }\end{array}$ & PI & IC50 CY & IA1 (mM) & $\overline{\mathrm{IC} 50 \mathrm{CYH}}$ & $9 \mathrm{~A} 1(\mathrm{mM})$ & $\begin{array}{c}\text { IC50 phLM } \\
(\mathrm{mM})\end{array}$ & $\begin{array}{c}\text { SI } \\
(1 \mathrm{~B} 1: 1 \mathrm{~A} 1)\end{array}$ & $\begin{array}{c}\text { SI } \\
(1 \mathrm{~B} 1: 19 \mathrm{~A} 1)\end{array}$ & $\begin{array}{c}\text { SI } \\
\text { (1B1:phLM) }\end{array}$ \\
\hline & dark & light $^{a}$ & & dark & light ${ }^{a}$ & dark & light $^{\mathrm{a}}$ & dark light & & & \\
\hline 1 & 0.083 & nd & - & 1.43 & nd & $>30$ & nd & 10.98 nd & 17.2 & $>360$ & 132 \\
\hline 2 & 0.057 & nd & - & 10.0 & nd & 10.71 & nd & 1.18 & 175 & 188 & 16 \\
\hline 3 & 0.00031 & nd & - & 4.57 & nd & 14.42 & nd & $0.334 \mathrm{nd}$ & 14,700 & 46,500 & 1,077 \\
\hline 4 & 0.19 & 0.012 & 15.8 & $\sim 30$ & nd & 13.27 & nd & $22.48 \quad 0.92$ & $\sim 158$ & 70 & 77 \\
\hline 5 & 1.73 & 0.017 & 102 & 40.9 & 7.82 & 28.3 & 11.2 & $>30 \sim 30$ & 460 & 660 & $\sim 1,750$ \\
\hline 6 & 1.9 & 0.0003 & 6,333 & $>30$ & $>30$ & $>30$ & 18.84 & $\sim 30 \quad 1.28$ & $>100,000$ & 62,800 & 4,300 \\
\hline
\end{tabular}

a With $660 \mathrm{~nm}$ light, $58.7 \mathrm{~J} / \mathrm{cm}^{2}$

A stable HEK cell line was created with CYP1B1expression under the control of tetracycline to facilitate controlled and titratable expression (see the SI for more details). A cell line was also created for CYP1A1 as a counter screen to evaluate selectivity. CYP1A1 is the closest family member, and shares $38 \%$ sequence identity with CYP1B1. ${ }^{28}$ The fluorogenic substrate, 7-ethoxyresorufin (REE), is a validated substrate for both CYPs and was used in both assays. Cytochrome P450 oxidoreductase (POR) is the required reductase partner for CYPs, and was also overexpressed in these systems to improve catalytic efficiency and the resulting signal for the assay.

Gratifyingly, both 2 and $\mathbf{3}$ were potent and selective inhibitors of CYP1B1 (Table 1, Figure 1b and c). While TMS exhibited an $\mathrm{IC}_{50}$ (the concentration for $50 \%$ inhibition) of $83 \mathrm{nM} 2$ was similarly potent, at $57 \mathrm{nM}$. Compound 3 was far superior, with an $\mathrm{IC}_{50}$ of 390 pM, making it one of the most potent CYP1B1 inhibitors reported. It was also remarkably selective, providing a selectivity index (SI) value of $>14,000$ for CYP1B1 vs. 1A1. Given the SI of 17 for TMS, this demonstrates that coordinating inhibitors can provide a $\sim 1,000$-fold improvement in selectivity.

As CYP19A1 metabolizes testosterone to estradiol, and estradiol is a known substrate of CYP1B1, we reasoned that the two enzymes might share features in their active sites that could result in overlapping 
inhibition profiles by the new inhibitors. Thus, the activity against CYP19A1 was evaluated using a recently developed in-cell fluorometric assay. ${ }^{29}$ While there was a small loss in selectivity for compound 2 compared to 1 , the pyridyl substituent in $\mathbf{3}$ improved the SI values over $\mathbf{1}$ by $>100$-fold.

The cellular assay results demonstrate that the inhibitors were obviously cell penetrant. Regular evaluation over $48 \mathrm{hrs}$ demonstrated the cells were healthy and their growth rates were unaffected. There were no observed modifications in cellular morphology, and it was concluded that the compounds did not induce damaging off-target effects.

Pooled human liver microsomes were used to test the potential impact of the inhibitors on multiple polymorphisms of highly promiscuous, drug metabolizing CYPs. ${ }^{30}$ There are more than 10 different CYPs in this preparation, and the use of pooled microsomes ensures that genetic variability in CYPs is represented. ${ }^{31}$ While 2 was less selective than TMS, likely due to the presence of a coordinating pyrimidine, inhibitor $\mathbf{3}$ was $>1,000$-fold selective for CYP1B1 (Figure 1c). This is notable, as the liver CYPs have evolved to bind the widest possible variety of molecules.

To provide context, we compared these results to those reported for anastrozole, a drug used by breast cancer patients for durations of 5-10 years as a maintenance therapy. Anastrozole is at least 500 -fold selective for CYP19A1 over liver CYPs; ${ }^{32}$ this reflects an upper limit for extremely selective CYP inhibitors. In contrast, CYP inhibitors such as ketoconazole are used with great caution, as their selectivity is poor. Ketoconazole's selectivity for its target, fungal CYP51, is only 2-fold compared to the human isoform of CYP51 ( $\mathrm{K}_{\mathrm{d}}$ values of 27 and $\left.42 \mathrm{nM}\right),{ }^{33}$ and it is a potent inhibitor of CYP3A4 $\left(\mathrm{IC}_{50}=40 \mathrm{nM}\right),{ }^{34}$ as well as members of the 1B, 2B and $2 \mathrm{C}$ families. ${ }^{35}$ Thus, the novel CYP1B1 inhibitors reported here provide SI values that rival or exceed the most selective CYP inhibitors used in the clinic.

Computational studies. The closely related structures of TMS, $\mathbf{2}$, and $\mathbf{3}$, but their wide range of potencies in CYP1B1 motivated computational studies to rationalize structure-activity relationships. However, simple molecular docking experiments were unable to identify significant differences in the protein-ligand complex, as the top predicted binding poses overlayed with a calculated RMSD of only $0.1 \AA$, as shown in Figure 1a. Short molecular dynamics trajectories were completed, and while some crucial residue contacts are shared, compounds $\mathbf{2}$ and $\mathbf{3}$ diverge in their behavior over the 20 ns trajectories. Compound 3 immediately produced a relatively stable RMSD of $1.67 \AA$, while 2 exhibited wide fluctuations for the first half of the trajectory before converging on a semi-stable binding pose at an RMSD of $2.52 \AA$ (Figure S1). The more dominant interactions in the trajectory of $\mathbf{3}$ include hydrophobic contacts with Val126 and Ala330, $\pi$-stacking with Phe134, and the formation of a water bridge with Ser127 (Table S1). Key interactions in the trajectory of 2 include the formation of transient water bridges with Gly329, Thr334, and a mixture of water bridge formation and hydrophobic contacts with lle399. Both molecules engage in $\pi$-stacking interactions with Phe231 and Phe268 with comparable duration. However, differences in pKa values for methyl pyrimidine vs. methyl pyridine (2.0 vs. 5.9$)$ may provide a partial explanation for the variation in activity, as the greater basicity of $\mathbf{3}$ should result in a stronger coordinative bond, and thus, improved potency. ${ }^{36}$ 
Mutational studies. Site directed mutagenesis was used to probe the importance of specific active-site contacts identified through the structural assessment (Figure 2a, b). Both TMS (1) and a-naphthoflavone (ANF), a polyaromatic CYP1 family inhibitor, were used as controls. While ANF binding is dominated by hydrophobic contacts, $\mathbf{1}$ was anticipated to be able to engage in some polar interactions. In all cases, conservative mutations were made to maintain polar or hydrophobic character and approximate size. Notably, the Phe231Leu mutant was inactive. This supported the premise that Phe231 is responsible for orienting and stabilizing contacts with small molecules, as this interaction would be essential for the binding of the 7-ethoxyresorufin substrate. All the other mutants created were active, and exhibited a range of $\mathrm{IC}_{50}$ values with the different inhibitors, as shown in Table S2. The ratio of $\mathrm{IC}_{50}$ values for the WT vs. the mutant are shown in Figure 2c, and reflects the importance of the specific contact. The Ser269Ala mutation, located in the $\mathrm{G}$ helix, had relatively little effect. The Phe134Leu mutant, which eliminated a predicted edge-to-face aromatic interaction with inhibitors, had a moderate impact. This highly conserved sidechain, analogous to Phe123 in CYP1A1, is located on the interior face of the active site and is also implicated in orientation of substrates.

The mutations that had the largest impact on inhibitor potency were Ser127Ala, GIn332Glu, and Asp333Asn, with a decrease in activity of 2-3 orders of magnitude. The Ser127 residue is located in the upper corner of the active site, on the B-C loop. It is not expected to directly contact the inhibitors, but forms a hydrogen bond with Asp326 of the I helix, and thus may impact the position of this helix with regards to the B-C loop. The Asp326 sidechain also engages in a H-bond with ANF, which may be strengthened by the replacement of the Ser127 with an alanine. This type of interaction could also explain the surprising increase in potency for compound 1 . However, the mutation has the opposite effect for $\mathbf{2}$ and $\mathbf{3}$, demonstrating a change in the molecular factors regulating their binding.

Residues GIn332Glu and Asp333Asn are located on the I helix, which transverses the active site and substrates and inhibitors rest against. Asp333 forms a salt bridge with Lys512 of b sheet 4 to regulate tertiary structure; this interaction is conserved in the CYP1 family. In contrast, a phenylalanine is found in CYP 1A1 in the position analogous to GIn332. The importance of the contacts made between $\mathbf{2}$ and $\mathbf{3}$ and these amino acids may be the key to the extraordinary selectivity observed for these inhibitors for CYP1B1 over CYP1A1.

Synthesis of photocaged CYP1B1 inhibitors. A variety of Ru(II) scaffolds were recently synthesized and assessed in order to identify a suitable inorganic system that was effective for photocaging enzyme inhibitors. ${ }^{37}$ One structure provided the desired biocompatibility, thermal stability, and ability to be triggered with visible light (from $450-660 \mathrm{~nm}$; Scaffold II, Chart 1). This Ru(II) photocage incorporated a $2,2^{\prime}$-biquinoline ligand, which shifted the absorption profile to longer wavelengths. ${ }^{37,38}$ This shift is due to the lower energy of the metal to ligand charge transfer (MLCT) transitions, which depend on the lowest unoccupied molecular orbital (LUMO) of the conjugated bibiquinoline ligand; due to the extended conjugation of quinoline, the transition is bathochromically shifted from analogous 2,2'-bipyridyl systems. The use of longer wavelengths of light are appealing for the ability to achieve greater depths of 
penetration into tissues, but it was also an important design feature for the complexes, as stilbene systems undergo trans to cis photoisomerizations with high energy (generally UV) light. ${ }^{39}$

Additional optimization included incorporation of carboxylic acids to the biquinoline. The [2,2'biquinoline]-4,4'-dicarboxylic acid ligand (also known as bicinchoninic acid, bca) reduced cellular toxicity caused by the metal complex, such that no adverse effects were observed up to $100 \mathrm{mM}$ concentrations. ${ }^{37}$ To satisfy three of the remaining coordination sites in the octahedral complex, the tridentate 2,2';6',2"-terpyridine (tpy) ligand was added. This left one site available for coordination of the active inhibitor, which is a monodentate ligand. The prodrug model systems containing pyridine and pyrimidine were stable in media and non-toxic with light exposure, validating the use of the $\mathrm{Ru}(\mathrm{II})$ scaffold for photocages.

Compounds $\mathbf{2}$ and $\mathbf{3}$ were combined with Scaffolds I and II to create photocaged CYP1B1 inhibitors, resulting in octahedral Ru(II) polypyridyl coordination complexes 4-6 (Chart 1). These structures are contrasted to previous photocaged enzyme inhibitors designed using the $\mathrm{Ru}(\mathrm{bpy})_{2}$ scaffold (bpy=2,2'bipyridyl), ${ }^{9,40-42}$ which allows for incorporation of one or two inhibitors for each molecular component. While combinations of tridentate ligands and strain-inducing bidentate ligands creates stoichiometric photocages, the advantage is that these systems have more predictable photochemistry. ${ }^{43}$

Table 2. Thermal stability, photophysical, and photochemical properties of compounds 4-6.

\begin{tabular}{|c|c|c|c|}
\hline Cmpd & $\lambda$ abs, $\mathrm{nm},(\varepsilon(\mathrm{M}-1 \mathrm{~cm}-1))$ & FPS & Stability $^{\mathrm{a}}$ \\
\hline$\overline{4}$ & $395(23,900) ; 455(15,800) ; 535(10,900)$ & $0.074 \pm 0.002 \mathrm{~b}$ & 47.7 \\
\hline 5 & $400(13,000) ; 455(8,300) ; 540(6,300)$ & $0.033 \pm 0.003^{b}$ & 67.0 \\
\hline $6^{\mathrm{d}}$ & $380(21,500) ; 455(7,300) ; 550(6,600)$ & $\begin{array}{l}0.0075 \pm 0.0003^{b} \\
0.0081 \pm 0.0006^{c}\end{array}$ & 98.6 \\
\hline
\end{tabular}

Determined as \% remaining at $24 \mathrm{~h}\left(37^{\circ} \mathrm{C}\right)$ in H2O. ${ }^{\mathrm{b}}$ Calculated by optical approach. ${ }^{\mathrm{C}}$ Calculated by HPLC approach. ${ }^{\mathrm{d}}$ FPS for ompound 6 was determined in MeCN.

Evaluation of Ru(II) photocages. As shown in Figure S2, the absorption profiles of complexes 4-6 varied as a function of the monodentate "caged" ligand (compound $\mathbf{5}$ vs $\mathbf{6}$ ) and the bidentate ligands (compound 4 vs 5). Both mono- and bidentate ligands had an impact on the longest wavelength absorption peak, $\lambda_{\max }$ and extinction coefficient $(\varepsilon)$ values (Table 2). Complexes 4- $\mathbf{6}$ exhibit maxima between $\sim 530$ and $545 \mathrm{~nm}$ in $\mathrm{H}_{2} \mathrm{O}$, with tailing absorption out to $650 \mathrm{~nm}$. The coordination of the 4substituted pyridyl ligand induced a bathochromic shift of $15 \mathrm{~nm}$, and a tail that extended to $700 \mathrm{~nm}$, facilitating activation with low energy light (Figure 3a). 
In order to standardize photochemical evaluation and to compare the photosubstitution for 46 with reference compounds studied previously, the quantum yield for photosubstitution, $\Phi_{\mathrm{PS}}$, was determined in $\mathrm{H}_{2} \mathrm{O}$ using $470 \mathrm{~nm}$ light. As anticipated, variation in yields was observed, with $\Phi_{\mathrm{PS}}$ ranging from 0.008 to 0.074 (Table 2). The coordination of pyrimidine-based ligand (3) to $\mathrm{Ru}(\mathrm{II})$ scaffolds (compound 4 and 5) resulted in 4-10 fold higher $\Phi_{\mathrm{PS}}$ compared with complex $\mathbf{6}$. This is in an agreement with the photochemical features of $\mathrm{Ru}(\mathrm{II})$ complexes with unsubstituted pyridine and diazines as monodentate ligands. ${ }^{37}$

The stability of each complex was assessed over 24 hours under aqueous conditions at $37^{\circ} \mathrm{C}$. The compounds with the higher $\Phi_{\mathrm{PS}}$ (4 and $\mathbf{5}$ ) exhibited slow degradation over $24 \mathrm{hr}$, while complex 6 remained stable, with less than $2 \%$ degradation (Fig. S7). Given the potency of the caged inhibitor, thermal stability is of critical importance, making complex 6 the preferred photocaged candidate.

Photoactivated CYP inhibition. Both compounds $\mathbf{4}$ and $\mathbf{5}$ were effective in providing photocontrol over inhibitor delivery, with photoactivity indices ( $\mathrm{Pl}$; the ratio of the $\mathrm{IC}_{50}$ values in the light and dark) of 16$102\left(l_{\text {ex }}=660 \mathrm{~nm}\right)$. These results were highly promising for the complexes as photocaged enzyme inhibitors. However, the potency of the intact complexes (0.2-2 $\mathrm{mM}$ ) was striking. As the experiments were performed in a cell-based assay, inhibition of CYP1B1 as a result of binding to the protein in a nonspecific manner was ruled out. It was plausible that the caged inhibitor interacts with some important surface region, such as the P450 oxidoreductase binding site. As all CYPs share the same fold, this could result in non-specific inhibition of other CYPs. However, compounds $\mathbf{4}$ and $\mathbf{5}$ had no impact on CYP19A1 or CYP1A1 at concentrations ${ }^{3} 20 \mathrm{mM} ; \mathrm{Ru}(\mathrm{bpy})_{3}$ also did not inhibit CYP1B1. Thus, the engagement with the enzyme is selective both with regards to the enzyme target and the presence of the inhibitors in the $\mathrm{Ru}(\mathrm{II})$ structure.

The best photocaged CYP1B1 inhibitor, compound $\mathbf{6}$, was created by incorporation of $\mathbf{3}$ into scaffold II. The complex could be activated with low energy light (Figure 3a), cleanly ejected $\mathbf{3}$ (Figure 3b, c) and produced complex 7, which was biologically inert. As with $\mathbf{4}$ and $\mathbf{5}$, the intact complex exhibited inhibition of CYP1B1, but had little effect on other CYPs up to concentrations of $30 \mathrm{mM}$ (Figure 2d, Table 1). Photoremoval of the $\mathrm{Ru}(\mathrm{II})$ protecting group with $660 \mathrm{~nm}$ light resulted in an $\mathrm{IC}_{50}$ of $300 \mathrm{pM}$ for inhibition of CYP1B1, which corresponded to a PI value of $>6,300$. This represents the largest PI value for any $\mathrm{Ru}(\mathrm{II})$ photocage, by $10-1,000$-fold, depending on the system. We are not aware of other inorganic or organic photocages that provide this level of selectivity.

The best combination of co-ligands used in the Ru(II) photocage is vital to the creation of biocompatible systems that can be activated with low energy light. Addition of carboxylic acids to the biquinoline ligand reduced inhibition of the CYP1B1 enzyme by the complex by $\sim 10$-fold (complex $\mathbf{5}$ vs $\mathbf{4}$ ), and concentrations ${ }^{3} 30 \mathrm{mM}$ were needed to observe interactions with other CYPs. However, to achieve $>10^{2}-$ fold differences in activity based on photocontrol required the creation of more potent inhibitors, with activity at low- to sub-nM concentrations. We believe this may be a consistent feature for light-activated 
P450 inhibitors until a photoactive Ru(II) scaffold is found that is biologically inert and does not allow for interactions with proteins.

Impact on protein stability. To probe the impact of these inhibitors on the biophysics of CYP1B1, the thermal stability of the recombinant enzyme was assessed and compared with ANF. Circular Dichroism (CD) was used to monitor changes in the secondary structure with increasing temperature. ANF is known to bind with its flat surface resting against the helix I between Gly329 and Ala330, and p-stacked with Phe231. ${ }^{28}$ The thermal stability of CYP1B1 shifted by $3{ }^{\circ} \mathrm{C}$ with $10 \mathrm{mM}$ ANF; a $2{ }^{\circ} \mathrm{C}$ stabilization was observed with inhibitor $\mathbf{3}$ (Table 3, Fig. S20). No shift in stability was seen with 6 at concentrations up to $20 \mathrm{mM}$. Thus, while the intact metal complex is able to inhibit the enzyme, it is not binding in a manner that enhances stability.

These findings, as well as those of others', ${ }^{11}$ clearly demonstrate that large $\mathrm{Ru}(\mathrm{II})$ complexes are able to inhibit CYPs. Indeed, efforts to develop photoactive Ru(II) systems for inhibition of another CYP, CYP3A4, were unsuccessful, with the intact complex often having greater activity than the released inhibitor. This present study establishes that this inhibition occurs with notable selectivity in cells, despite the presence of many competing biomolecules.

We demonstrate with this work a strategy whereby CYP engagement can be reduced through molecular design of both the photocage and the inhibitor. However, we have not yet found a Ru(II) photocage scaffold that completely eliminates the inhibition of the target CYP. As anticipated, given their size, complexes 4, 5, and $\mathbf{6}$ could not be docked into the CYP1B1 structure, so rational design to reduce binding is not possible. What remains to be determined is what drives the interaction of metal-coordinated CYP inhibitors with such extraordinary affinity and selectivity to their target enzymes. As the metal center blocks key components of the inhibitor, and the enzyme must distort into an thermodynamically unfavored conformation to accommodate this unnatural system, these metal complexes for us to reassess what we assume about CYP inhibitors.

\section{Discussion}

Creation of selective, stable, and responsive agents for photopharmacology is quite challenging. Photoswitching compounds, which provide the ability to reversibly turn "on" and "off" the activity of a variety of molecules, thereby enabling dynamic studies, commonly exhibit $\sim 10$-fold differences between their active and inactive forms. ${ }^{44,45}$ Photocages, which are irreversible, tend to have higher PI values, but the best organic systems usually require cell damaging high energy light, and PI values remain below 100. Inorganic systems provide advantages that include use of longer wavelengths of light, and $\mathrm{Ru}(\mathrm{II})$ has emerged as the most promising protecting group. ${ }^{41,46,47}$ In a recent report, five different light activated $\mathrm{Ru}(\mathrm{II})$ complexes containing CYP3A4 inhibitors were designed and evaluated, and the highest PI value achieved was 2.1, highlighting the difficulty of this goal. ${ }^{11}$ This is a persistent problem for $\mathrm{Ru}(\mathrm{II})$ photocages, with dark $\mathrm{IC}_{50}$ values commonly being in the $\mathrm{mM}$ range. For such systems, $\mathrm{PI}$ values are 
generally below 40 , including for caged inhibitors of cysteine proteases, ${ }^{40,48,49}$ nicotinamide phosphoribosyltransferase (NAMPT) ${ }^{50}$ tubulin polymerization, ${ }^{51}$ and CYPs. ${ }^{10}$

In this study, we have achieved unprecedented photocontrol for selective inhibition of CYP1B1. Rational redesign of CYP1B1 inhibitors resulted in a new compound with $\mathrm{PM} \mathrm{IC} \mathrm{C}_{50}$ for CYP1B1 and selectivity over other CYPs of 3-4 orders of magnitude. This molecule, combined with an optimized Ru(II) caging scaffold, yielded the best system, which possessed protein inhibition at picomolar concentration when irradiated with red light. To the best of our knowledge, $\mathbf{3}$ is the most selective reported CYP inhibitor, and 6 provides the highest photoactivity index described for inhibition of a protein by a $\mathrm{Ru}(\mathrm{II})$ complex.

\section{Methods}

Full and detailed experimental protocols can be found in the Supplementary Information.

\section{Synthesis}

General. All materials were purchased from commercial sources and used without any further purification. All $1 \mathrm{H}-\mathrm{NMR}$ and 13C-NMR were obtained on a Varian Mercury spectrometer $(400,100 \mathrm{MHz})$ and chemical shifts are reported relative to the residual solvent peaks. Electrospray ionization (ESI) mass spectra were obtained on a Varian 1200L mass spectrometer at the Environmental Research Training Laboratory (ERTL) at the University of Kentucky. UV/Vis absorption spectra were obtained on a BMG Labtech FLUOstar Omega microplate reader. Light activation experiments were performed using a 470 $\mathrm{nm}$ and $660 \mathrm{~nm}$ LED array from Elixa. The Prism software package was used to analyze data.

HPLC analysis for purity and photoejection products. The purity of $\mathrm{Ru}(\mathrm{II})$ complex and photoejection products were analyzed using an Agilent 1100 Series HPLC equipped with a model G1311A quaternary pump, G1315B UV diode array detector and Chemstation software version B.01.03. Chromatographic conditions were optimized on a Column Technologies Inc. C18(2), $100 \AA$ (250 mm × $4.6 \mathrm{~mm}$ inner diameter, $5 \mu \mathrm{M})$ fitted with a Phenomenex C18 $(4 \mathrm{~mm} \times 3 \mathrm{~mm})$ guard column. Injection volumes of $20 \mu \mathrm{L}$ of $30-100 \mu \mathrm{M}$ solutions of the complex were used. The detection wavelength was $280 \mathrm{~nm}$. Mobile phases were: mobile phase $\mathrm{A}, 0.1 \%$ formic acid in $\mathrm{dH} 2 \mathrm{O}$; mobile phase $\mathrm{B}, 0.1 \%$ formic acid in HPLC grade acetonitrile. The mobile phase flow rate was $1.0 \mathrm{~mL} / \mathrm{min}$. The following mobile phase gradient was used: 98-95\% A (containing 2-5\% B) from 0 to $5 \mathrm{~min} ; 95-70 \% \mathrm{~A}(5-30 \% \mathrm{~B}$ ) from 5 to $15 \mathrm{~min} ; 70-40 \% \mathrm{~A}$ (30-60\% B) from 15 to $20 \mathrm{~min} ; 40-5 \%$ A (60-95\% B) from 20 to $30 \mathrm{~min} ; 5-98 \% \mathrm{~A}(95-2 \% \mathrm{~B})$ from 30 to $35 \mathrm{~min}$; reequilibration at $98 \% \mathrm{~A}(2 \% \mathrm{~B})$ from 35 to $40 \mathrm{~min}$.

Counter-ion exchange. Prior to photoejection studies and biological testing, counterion exchange was performed on compound 4 . The $\mathrm{PF}_{6}{ }^{-}$salt of $\mathbf{4}$ was converted to the $\mathrm{Cl}^{-}$salt by dissolving $10 \mathrm{mg}$ of product in $2 \mathrm{~mL}$ methanol. The dissolved product was loaded onto an Amberlite IRA-410 chloride ion exchange column, eluted with methanol, and the solvent was removed in vacuo. 
Photoejection studies. Quantum yields for the complexes 4-6 were determined by optical and HPLC approaches as described previously. ${ }^{27,37}$ The $\mathrm{Ru}(\mathrm{II})$ complexes were analyzed in a 96 well plate at a final concentration of $30-50 \mu \mathrm{M}$ and a path length of $0.5 \mathrm{~cm}$. Scans were taken at set time points for 240 minutes. In all cases, the light source was a $470 \mathrm{~nm}$ LED array from Elixa. The photon flux of the lamp for irradiation in the plate was determined by ferrioxalate actinometer $(1.77 \mathrm{E}-8 \mathrm{E} / \mathrm{s})$. The absorbance of complexes at $470 \mathrm{~nm}$ were from 0.12 to 0.36 , with photon absorption probability (F) from 0.22 to 0.42 . Therefore, the moles of photon absorbed have been calculated as the product of photons irradiated and photon absorption probability.

The quantum yield for photosubstitution of complex 6 in MeCN was calculated based on HPLC analysis, and compared with the optical approach. The Ru(II) complex was irradiated in a quartz cuvette at a final concentration of $40 \mu \mathrm{M}$ and a path length of $1 \mathrm{~cm}$. The photon flux of the lamp for irradiation in cuvette was determined by ferrioxalate actinometer $(2.32 \mathrm{E}-7 \mathrm{E} / \mathrm{s})$. The absorbance of complex at a concentration of $40 \mu \mathrm{M}$ at $470 \mathrm{~nm}$ was 0.27 with photon absorption probability $(\mathrm{F})$ of 0.46 .

\section{Enzyme activity studies.}

In cell activity assays for CYP1B1, 1A1, and 19A1: The genes for CYP1B1, CYP1A1 and CYP19A1 were purchased from Origene. To allow for maximal turnover, the pcDNA4 T/O vector was modified for dual expression with P450 oxidoreductase (POR). Both the CYP and POR expression was under the control of the Tet02 inducible CMV promoter.2 In brief, CYP1B1, CYP1A1 or CYP19A1 was cloned into pcDNA4 T/0. Agel and BsiWI restriction sites were then incorporated into the plasmids. The promoter region from pcDNA4 T/O was amplified and ligated creating a second TetO2 inducible CMV promoter with the pcDNA4 T/O multiple cloning sites (MCS). POR was cloned into this MCS using the Kpnl and Xhol restriction sites.

Cell lines were generated in the HEK293 T-Rex cell line. Following transfection, cells were selected with $500 \mu \mathrm{g} / \mathrm{ml}$ Zeocin, and $7.5 \mu \mathrm{g} / \mathrm{ml}$ Blasticidin to create a stable pool. Inducible expression of CYP and POR was confirmed by immunoblot. The cell lines were maintained in DMEM supplemented with $10 \%$ FBS, $100 \mathrm{U}$ Penicillin and $100 \mu \mathrm{g} / \mathrm{ml}$ Streptomycin at $37^{\circ} \mathrm{C}$ with $5 \% \mathrm{CO} 2$.

Cell lines were seeded onto Geltrex coated 96 well plates at 40,000 cells/well, and grown overnight in DMEM media containing $1 \mu \mathrm{g} / \mathrm{ml}$ tetracycline. The media was then replaced with Opti-MEM supplemented with $2 \%$ FBS and $1 \mu \mathrm{g} / \mathrm{ml}$ tetracycline. Compounds were serially diluted in Opti-MEM supplemented with $2 \% \mathrm{FBS}$ and $1 \mu \mathrm{g} / \mathrm{ml}$ tetracycline and an equal volume of compound added to the cells. Following a $1 \mathrm{hr}$ incubation, resorufin ethyl ether (REE; for CYP1B1 and 1A1) or dibenzylfluorescein (DBF; for 19A1) was added to the cells for a total concentration of $5 \mu \mathrm{M}$ for REE and $1 \mu \mathrm{M}$ for DBF. Time points were taken over a period of 24 hrs using a Spectrafluor Plus plate reader (Tecan) with an excitation wavelength of $530 \mathrm{~nm}$ and emission wavelength of $595 \mathrm{~nm}$ for CYP1B1 and CYP1A1, and excitation wavelength of $480 \mathrm{~nm}$ and emission wavelength of $530 \mathrm{~nm}$ for CYP19A1. 
For the light activated compounds, the experiment was modified as follows: Cells were seeded and grown overnight as described above. Media was replaced with L-15 containing $1 \mu \mathrm{g} / \mathrm{ml}$ tetracycline. Compounds were serially diluted in L-15 with $1 \mu \mathrm{g} / \mathrm{ml}$ tetracycline and added to the cells followed by a $1 \mathrm{hr}$ incubation. The cells were then exposed to red light for 1 hour $\left(660 \mathrm{~nm}, 58.7 \mathrm{~J} / \mathrm{cm}^{2}\right)$ followed by the addition of an equal volume of Opti-MEM supplemented with $4 \% \mathrm{FBS}$ and $1 \mu \mathrm{g} / \mathrm{ml}$ tetracycline. REE or DBF was added as described above.

Analysis in pooled human live microsomes ( $p h L M$ ): Compounds were screened for inhibition of cytochrome P450 enzymes in human liver microsomes using a 96 well kinetic assay. The final concentration of DMSO did not exceed $0.8 \%$. Compound stocks were diluted in dose response, and added in $100 \mathrm{mM}$ potassium phosphate buffer $\mathrm{pH} 7.8$ with $50 \mu \mathrm{M}$ 7-benzoyloxy-4-trifluoromethylcoumarin (Corning, prepared as a $10 \mathrm{mM}$ DMSO stock) and $16 \mu \mathrm{g}$ of phLMs (XenoTech, prepared as $20 \mathrm{mg}$ protein/mL suspended in $250 \mathrm{mM}$ sucrose) per well. The compounds were incubated for $5 \mathrm{~min}$ in the absence of light at $25^{\circ} \mathrm{C}$. The 96 well plate was then either protected from light or irradiated with red light for $1 \mathrm{hr}\left(660 \mathrm{~nm}, 58.7 \mathrm{~J} / \mathrm{cm}^{2}\right)$. Enzymatic turnover was initiated upon addition of NADPH (TCl, prepared immediately prior to addition) at a concentration $1.2 \mathrm{mM}$ per well. To determine background fluorescence from the assay, NADPH was omitted from several control wells. The reactions were immediately placed in a SpectraFluor Plus plate reader (Tecan) preheated to $37^{\circ} \mathrm{C}$, and fluorescence measurements taken every $3 \mathrm{~min}$ for $120 \mathrm{~min}$ with excitation of $435 \mathrm{~nm}$ and emission of $525 \mathrm{~nm}$. Fluorescence measurements in the presence of NADPH were subtracted from those in the absence of NADPH, and the data was processed with GraphPad Prism 6. The linear portion of the signal for each dose point with respect to time was first fitted to a line to determine the rate. The resulting slope of these lines was then plotted against the logarithmic concentration of the study compound to provide a dose-response curve.

CYP1B1 stability. CYP1B1 was expressed and purified as reported. The purified enzyme exhibited the classical shift to the $450 \mathrm{~nm}$ form in the presence of carbon monoxide, and demonstrated spin shifts in the presence of inhibitors. The stability of CYP1B1 in the presence of several inhibitors was determined by temperature melt, with the change in ellipticity monitored at $230 \mathrm{~nm}$ by circular dichroism with a J-815 Spectrometer (Jasco). The enzyme was prepared at a concentration of $0.1 \mathrm{mg} / \mathrm{ml}$ in $20 \mathrm{mM}$ Potassium Phosphate pH 7.4, $300 \mathrm{mM} \mathrm{NaCl}, 10 \mathrm{mM}$ CHAPS, 20\% Glycerol with $1 \mathrm{mM}$ 2-mercaptoethanol. Inhibitors were incubated with the enzyme at $25{ }^{\circ} \mathrm{C}$ for $10 \mathrm{~min}$. Spectra were taken scanning from $200-260 \mathrm{~nm}$ in duplicate. The temperature was increased followed by a 3 min equilibration period before spectra acquisition. The ellipticity values at $230 \mathrm{~nm}$ were plotted against temperature and fit to determine the $T_{m}$ (Prism).

Table 3. $T_{m}$ values for recombinant CYP1B1 $+/$ - inhibitors 


\begin{tabular}{lll}
\hline Condition & Concentration & $\mathrm{T}_{\mathrm{m}}(\underline{\mathrm{o}} \mathrm{C})$ \\
\hline No compound & - & $48+/-0.80$ \\
ANF & $10 \mu \mathrm{M}$ & $51+/-0.51$ \\
\hline $\mathbf{3}$ & $10 \mu \mathrm{M}$ & $50+/-0.56$ \\
\hline $\mathbf{6}$ & $20 \mu \mathrm{M}$ & $47+/-0.84$ \\
\hline
\end{tabular}

Docking and MD experiments. For the computational investigation of CYP1B1, the PDB structure 3PM0 was used, which has the inhibitor a-naphthoflavone (ANF) bound in the active site. ${ }^{28} 52$ To prepare the published coordinates for computation, Maestro was used to process and refine the structure. The need for preparing protein crystal structures for computation is well established and includes manipulations not performed in the x-ray crystal structure refinement stage of data collection. ${ }^{53}$ These preparation steps include, but are not limited to, assignment of bond orders, addition of hydrogen atoms to the structure, optimization of the hydrogen bonding (H-bonding) network, resolution of atomic clashes, processing residues with missing electron density, and minimization of the protein structure. Zero-order bonds to metals were created, missing side chain $\mathrm{s}^{54}$ and loops ${ }^{55}$ were filled with Prime, and waters farther than 5 $\AA$ from heteroatom groups were removed. After this initial processing, the heme iron atom was set to a charge state of +3 to reflect the resting ferric form of the heme.

To test the ability of Maestro to reproduce key interactions, the bound ANF inhibitor was removed from the active site cavity, and then successfully docked using the Glide application ${ }^{56-58}$ to an RMSD of 0.34 angstroms from its original position.

To further evaluate the active site model for its ability to predict interactions, the CYP1 family substrate 7-ethoxyresorufin was docked into the CYP1B1. This molecule is the fluorogenic substrate used in the ethoxyresorufin-O-deethylase (EROD) assay, ${ }^{59}$ which relies on oxidative dealkylation to form resorufin as a measure of enzymatic activity. Docking results predict placement of the critical oxygen atom near the catalytic heme prosthetic group, while the $\pi$-system of the resorufin core is stabilized by nearby phenylalanine residues. These results support the fidelity of the active site model, and provide confidence in the predictive power of generated docking results.

Atomistic molecular dynamics (MD) simulations were employed to investigate how protein-ligand complexes evolve over time, and if this evolution highlights interactions not predicted by the static docking calculations. MD trajectories were set up and calculated using the Desmond package in Schrödinger's Maestro molecular modeling environment as follows. The input protein-ligand complex was taken from previously generated docking results, and the system was prepared for molecular dynamics using the system builder function and the OPLS3e force field. During this setup the simple point charge (SPC) solvent model was used to solvate an orthorhombic box with buffers of $10 \AA$ between the protein and any boundary of the box. The box volume was minimized, and chloride ions were added 
to neutralize the system. Chloride ions were excluded from placement within $10 \AA$ of the heme prosthetic group to prevent occupancy of the active site of the enzyme. This resulted in systems of approximately 50,000 atoms. These prepared systems were then loaded into the molecular dynamics task window and used for the calculation of trajectories with the following parameters. The simulation time was set to 20 ns with a recording interval of $1.2 \mathrm{ps}$ for the energy of the system and $20 \mathrm{ps}$ for the trajectory (atom positions) for a total of 1,000 frames recorded. An isothermal-isobaric (NPT) ensemble was used with a temperature of $300 \mathrm{~K}$ and a pressure of $1.01325 \mathrm{bar}$, and the model system was relaxed prior to simulation. Following simulation, analysis was completed using the simulation interactions diagram function within Maestro.

\section{Declarations}

Online content

Additional figures and tables.

Author contributions

E.C.G., D.K.H., and D.H. conceived the project, designed the experiments, and wrote the manuscript. A.C.H. performed experiments with phLMs. A.D.F. performed computational calculations. D.H. performed synthesis and photophysical measurements. D.K.H. created the assays, generated all mutants, and performed all biological and biophysical characterization. All authors analyzed the data, discussed the results and commented on the manuscript.

Acknowledgment

We gratefully acknowledge the support of the National Institute Of General Medical Sciences of the National Institutes of Health under Award Numbers R01GM138882 and 5R01GM107586.

Competing Interests

The authors declare no competing interests.

\section{References}

1. Manikandan, P. \& Nagini, S. Cytochrome P450 Structure, Function and Clinical Significance: A Review. Curr Drug Targets 19, 38-54, doi:10.2174/1389450118666170125144557 (2018).

2. Ma, C. X., Reinert, T., Chmielewska, I. \& Ellis, M. J. Mechanisms of aromatase inhibitor resistance. Nature Reviews Cancer 15, 261-275, doi:10.1038/nrc3920 (2015).

3. Guengerich, F. P. A history of the roles of cytochrome P450 enzymes in the toxicity of drugs. Toxicol Res, 1-23, doi:10.1007/s43188-020-00056-z (2020). 
4. Munkboel, C. H. et al. The classic azole antifungal drugs are highly potent endocrine disruptors in vitro inhibiting steroidogenic CYP enzymes at concentrations lower than therapeutic Cmax. Toxicology 425, 152247, doi:10.1016/j.tox.2019.152247 (2019).

5. Adis Medical, W. Long-term use of systemic azole antifungals can result in hepatotoxicity and other serious adverse effects. Drugs \& Therapy Perspectives 36, 112-115, doi:10.1007/s40267-019-00699-5 (2020).

6. Kitts, A., Phan, L., Ward, M. \& Holmes, J. B. in The NCBI Handbook [Internet]. 2nd edition (National Center for Biotechnology Information (US), 2014).

7. Foroozesh, M., Sridhar, J., Goyal, N. \& Liu, J. Coumarins and P450s, Studies Reported to-Date. Molecules 24, doi:10.3390/molecules24081620 (2019).

8. No organic photocages were found for CYP inhibitors in a search of the literature.

9. Zamora, A. et al. Ruthenium-containing P450 inhibitors for dual enzyme inhibition and DNA damage. Dalton Trans 46, 2165 - 2173, doi:10.1039/c6dt04405k (2017).

10. Li, A. et al. Illuminating cytochrome P450 binding: Ru(II)-caged inhibitors of CYP17A1. Chem. Commun. 53, 3673-3676, doi:10.1039/c7cc01459g (2017).

11. Toupin, N. et al. Photosensitive Ru(II) Complexes as Inhibitors of the Major Human Drug Metabolizing Enzyme CYP3A4. J Am Chem Soc 143, 9191-9205, doi:10.1021/jacs.1c04155 (2021).

12. Golbaghi, G. et al. Organoruthenium(II) Complexes Bearing an Aromatase Inhibitor: Synthesis, Characterization, in Vitro Biological Activity and in Vivo Toxicity in Zebrafish Embryos. Organometallics 38, 702-711, doi:10.1021/acs.organomet.8b00897 (2019).

13. Rubbiani, R. et al. In vivo Active Organometallic-containing Antimycotic Agents. RSC Chemical Biology, doi:10.1039/D1CB00123J (2021).

14. Area values calculated for $\mathrm{Ru}(\mathrm{tpy})(6,6$ 'dmbpy) and for $\mathrm{Ru}(\mathrm{typ})(\mathrm{bca})$.

15. Dunn, A. R., Dmochowski, I. J., Bilwes, A. M., Gray, H. B. \& Crane, B. R. Probing the open state of cytochrome P450cam with ruthenium-linker substrates. Proc Natl Acad Sci U S A 98, 12420-12425, doi:10.1073/pnas.221297998221297998 [pii] (2001).

16. Dunn, A. R. et al. Fluorescent probes for cytochrome p450 structural characterization and inhibitor screening. J Am Chem Soc 124, 10254-10255, doi:ja0271678 [pii] (2002).

17. Hays, A. M. et al. Conformational states of cytochrome P450cam revealed by trapping of synthetic molecular wires. J. Mol. Biol. 344, 455-469, doi:10.1016/j.jmb.2004.09.046 (2004). 
18. Glazer, E. C., Lee, Y. T., Wilson, R. F., Stout, C. D. \& Goodin, D. B. Three clusters of conformational states in p450cam reveal a multistep pathway for closing of the substrate access channel. Biochemistry 50, 693-703, doi:10.1021/bi101726d (2011).

19. Carrera, Alexa N., Grant, Marianne K. O. \& Zordoky, Beshay N. CYP1B1 as a therapeutic target in cardio-oncology. Clinical Science 134, 2897-2927, doi:10.1042/CS20200310 \%J Clinical Science (2020).

20. Horley, N. J. et al. Discovery and characterization of novel CYP1B1 inhibitors based on heterocyclic chalcones: Overcoming cisplatin resistance in CYP1B1-overexpressing lines. Eur. J. Med. Chem. 129, 159174, doi:10.1016/j.ejmech.2017.02.016 (2017).

21. Huang, R. S. et al. Genetic variants contributing to daunorubicin-induced cytotoxicity. Cancer Res 68, 3161-3168, doi:10.1158/0008-5472.CAN-07-6381 (2008).

22. Chang, I. et al. Loss of miR-200c up-regulates CYP1B1 and confers docetaxel resistance in renal cell carcinoma. Oncotarget 6, 7774-7787, doi:10.18632/oncotarget.3484 (2015).

23. De luliis, F., Salerno, G., Taglieri, L. \& Scarpa, S. Are pharmacogenomic biomarkers an effective tool to predict taxane toxicity and outcome in breast cancer patients? Literature review. Cancer Chemother Pharmacol 76, 679-690, doi:10.1007/s00280-015-2818-4 (2015).

24. Dumont, A. et al. ERCC1 and CYP1B1 polymorphisms as predictors of response to neoadjuvant chemotherapy in estrogen positive breast tumors. Springerplus 4, 327, doi:10.1186/s40064-015-1053-0 (2015).

25. Sissung, T. M. et al. Association of the CYP1B1*3 allele with survival in patients with prostate cancer receiving docetaxel. Mol Cancer Ther 7, 19-26, doi:10.1158/1535-7163.MCT-07-0557 (2008).

26. So-called Type II coordinating inhibitors are used in the clinic for CYP19A1 (anastrozole and letrozole), 17A1, CYP11B1 (metyrapone), and fungal CYP51 (including ketoconazole, fluconazole, itraconazole, and many others).

27. Havrylyuk, D., Deshpande, M., Parkin, S. \& Glazer, E. C. Ru(II) complexes with diazine ligands: electronic modulation of the coordinating group is key to the design of "dual action" photoactivated agents. Chem. Commun. 54, 12487-12490, doi:10.1039/c8cc05809a (2018).

28. Wang, A., Savas, U., Stout, C. D. \& Johnson, E. F. Structural characterization of the complex between alpha-naphthoflavone and human cytochrome P450 1B1. J Biol Chem 286, 5736-5743, doi:10.1074/jbc.M110.204420 (2011).

29. Heidary, D. K., Kriger, S. M., Hachey, A. C. \& Glazer, E. C. A Fluorometric CYP19A1 (Aromatase) Activity Assay in Live Cells. ChemMedChem, doi:10.1002/cmdc.202100326 (2021). 
30. Zhang, H. et al. Content and activity of human liver microsomal protein and prediction of individual hepatic clearance in vivo. Scientific Reports 5, 17671, doi:10.1038/srep17671 (2015).

31. Commercial preparations come from 50 individuals.

32. Grimm, S. W. \& Dyroff, M. C. Inhibition of Human Drug Metabolizing Cytochromes P450 by Anastrozole, a Potent and Selective Inhibitor of Aromatase. Drug Metabolism and Disposition 25, 598-602 (1997).

33. Hoobler, E. K. et al. Discovery of a novel dual fungal CYP51/human 5-lipoxygenase inhibitor: implications for anti-fungal therapy. PLoS One 8, e65928, doi:10.1371/journal.pone.0065928 (2013).

34. The reported IC50 is $40 \mathrm{nM}$. Two molecules of keotconozole are found in the crystal structure, and binding appears to be cooperative.

35. Sevrioukova, I. F. \& Poulos, T. L. Understanding the mechanism of cytochrome P450 3A4: recent advances and remaining problems. Dalton Transactions 42, 3116-3126, doi:10.1039/C2DT31833D (2013).

36. This hypothesis is related to the prevalence of the 1,2,4-triazole ring in CYP inhibitors, in contrast to the less basic 1,2,3-triazole ring. See K. P. Conner, P. Vennam, C. M. Woods, M. D. Krzyaniak, M. K. Bowman, W. M. Atkins, Biochemistry 2012, 51, 6441-6457.

37. Havrylyuk, D., Stevens, K., Parkin, S. \& Glazer, E. C. Toward Optimal Ru(II) Photocages: Balancing Photochemistry, Stability, and Biocompatibility Through Fine Tuning of Steric, Electronic, and Physiochemical Features. Inorg. Chem. 59, 1006-1013, doi:10.1021/acs.inorgchem.9b02065 (2020).

38. Wachter, E., Heidary, D. K., Howerton, B. S., Parkin, S. \& Glazer, E. C. Light-activated ruthenium complexes photobind DNA and are cytotoxic in the photodynamic therapy window. ChemComm 48, 96499651, doi:10.1039/c2cc33359g (2012).

39. Görner, H. \& Kuhn, H. J. in Advances in Photochemistry 1-117 (1994).

40. Respondek, T. et al. Light activation of a cysteine protease inhibitor: caging of a peptidomimetic nitrile with Ru(II)(bpy)2. J. Am. Chem. Soc. 133, 17164-17167, doi:10.1021/ja208084s (2011).

41. Zayat, L., Calero, C., Albores, P., Baraldo, L. \& Etchenique, R. A new strategy for neurochemical photodelivery: metal-ligand heterolytic cleavage. J. Am. Chem. Soc. 125, 882-883, doi:10.1021/ja0278943 (2003).

42. Zayat, L., Salierno, M. \& Etchenique, R. Ruthenium(II) bipyridyl complexes as photolabile caging groups for amines. Inorg. Chem. 45, 1728-1731, doi:10.1021/ic0512983 (2006). 
43. Bonnet, S., Collin, J.-P., Sauvage, J.-P. \& Schofield, E. Photochemical Expulsion of the Neutral Monodentate Ligand $\mathrm{L}$ in $\mathrm{Ru}\left(\mathrm{T}_{\mathrm{T}} \mathrm{rpy}^{*}\right)$ (Diimine)(L)2+: A Dramatic Effect of the Steric Properties of the Spectator Diimine Ligand. Inorg. Chem. 43, 8346-8354, doi:10.1021/ic0491736 (2004).

44. Donthamsetti, P. et al. Selective Photoswitchable Allosteric Agonist of a G Protein-Coupled Receptor. J. Am. Chem. Soc. 143, 8951-8956, doi:10.1021/jacs.1c02586 (2021).

45. Kolarski, D. et al. Photopharmacological Manipulation of Mammalian CRY1 for Regulation of the Circadian Clock. J. Am. Chem. Soc. 143, 2078-2087, doi:10.1021/jacs.0c12280 (2021).

46. Zayat, L., Filevich, O., Baraldo, L. M. \& Etchenique, R. Ruthenium polypyridyl phototriggers: from beginnings to perspectives. Philos Trans A Math Phys Eng Sci 371, 20120330, doi:rsta.2012.0330 [pii]10.1098/rsta.2012.0330 (2013).

47. Pérez, Y. R. \& Etchenique, R. Optical manipulation of animal behavior using a ruthenium-based phototrigger. Photochemical \& Photobiological Sciences 18, 208-212, doi:10.1039/c8pp00467f (2019).

48. Huisman, M. et al. Caging the uncageable: using metal complex release for photochemical control over irreversible inhibition. Chem Commun (Camb) 52, 12590-12593, doi:10.1039/c6cc07083c (2016).

49. Arora, K. et al. Catch and Release Photosensitizers: Combining Dual-Action Ruthenium Complexes with Protease Inactivation for Targeting Invasive Cancers. J Am Chem Soc 140, 14367-14380, doi:10.1021/jacs.8b08853 (2018).

50. Lameijer, L. N. et al. A Red-Light-Activated Ruthenium-Caged NAMPT Inhibitor Remains Phototoxic in Hypoxic Cancer Cells. Angew. Chem. Int. Ed. Engl. 56, 11549-11553, doi:10.1002/anie.201703890 (2017).

51. van Rixel, V. H. S. et al. Photo-Uncaging of a Microtubule-Targeted Rigidin Analogue in Hypoxic Cancer Cells and in a Xenograft Mouse Model. J Am Chem Soc 141, 18444-18454, doi:10.1021/jacs.9b07225 (2019).

52. Shimada, T. et al. Selectivity of polycyclic inhibitors for human cytochrome P450s 1A1, 1A2, and 1B1. Chem Res Toxicol 11, 1048-1056, doi:10.1021/tx980090+ (1998).

53. Sastry, G. M., Adzhigirey, M., Day, T., Annabhimoju, R. \& Sherman, W. Protein and ligand preparation: parameters, protocols, and influence on virtual screening enrichments. J Comput Aided Mol Des 27, 221234, doi:10.1007/s10822-013-9644-8 (2013).

54. Jacobson, M. P., Friesner, R. A., Xiang, Z. \& Honig, B. On the Role of the Crystal Environment in Determining Protein Side-chain Conformations. Journal of Molecular Biology 320, 597-608, doi:10.1016/s0022-2836(02)00470-9 (2002). 
55. Jacobson, M. P. et al. A hierarchical approach to all-atom protein loop prediction. Proteins 55, 351367, doi:10.1002/prot.10613 (2004).

56. Friesner, R. A. et al. Glide: a new approach for rapid, accurate docking and scoring. 1. Method and assessment of docking accuracy. J Med Chem 47, 1739-1749, doi:10.1021/jm0306430 (2004).

57. Halgren, T. A. et al. Glide: a new approach for rapid, accurate docking and scoring. 2. Enrichment factors in database screening. J Med Chem 47, 1750-1759, doi:10.1021/jm030644s (2004).

58. Friesner, R. A. et al. Extra precision glide: docking and scoring incorporating a model of hydrophobic enclosure for protein-ligand complexes. J Med Chem 49, 6177-6196, doi:10.1021/jm051256o (2006).

59. Burke, M. D. \& Mayer, R. T. Ethoxyresorufin: direct fluorimetric assay of a microsomal O-dealkylation which is preferentially inducible by 3-methylcholanthrene. Drug Metab Dispos 2, 583-588 (1974).

\section{Charts}

Chart 1 is in the supplementary files section.

\section{Figures}


A)

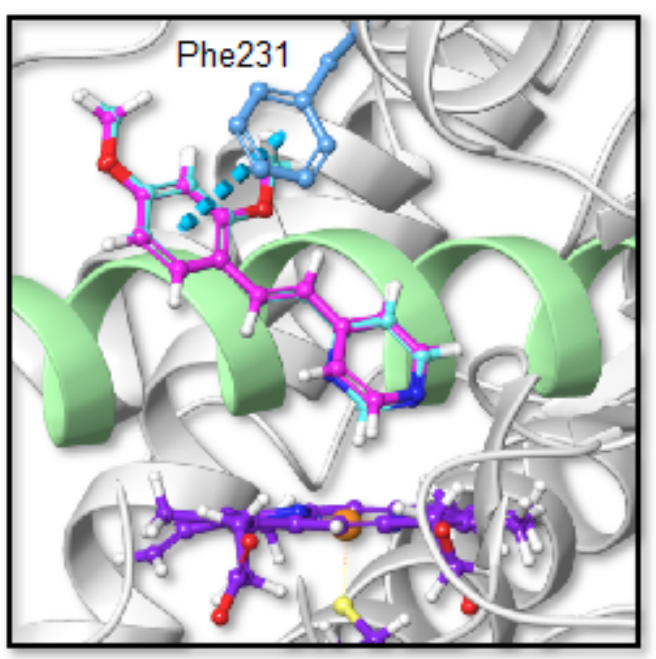

B)

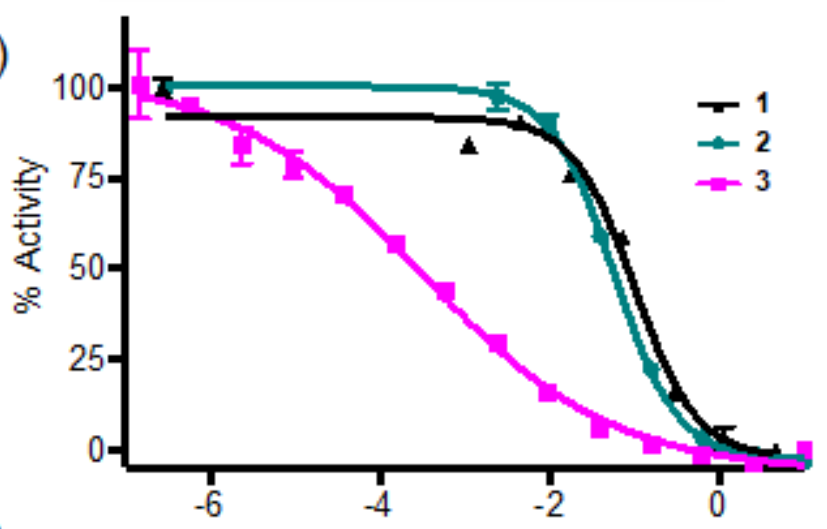

C)

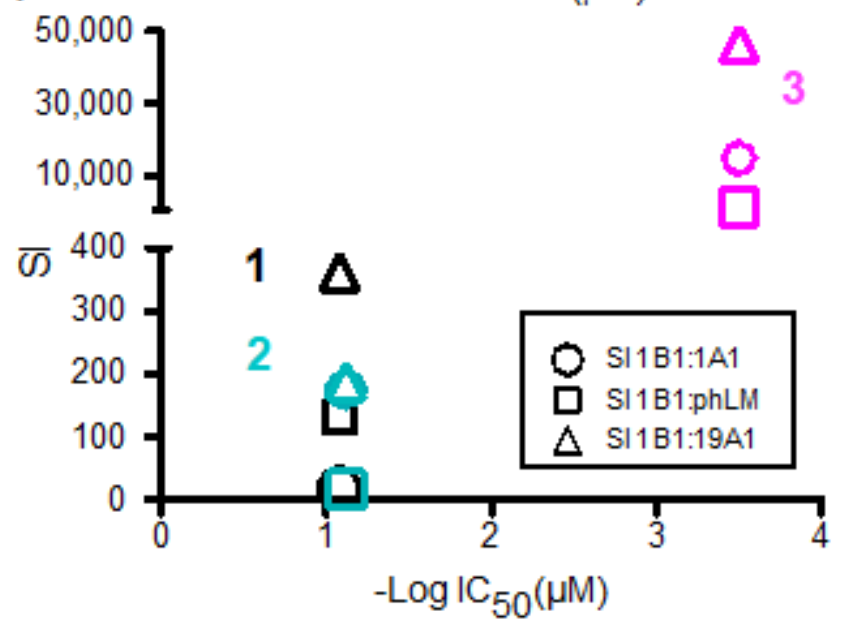

Figure 1

Heterocycle containing inhibitors for CYP1B1. a, Docking of 2 and 3 shows the position of the heterocycle over the heme (purple) and key contacts with Phe231. Docking based on PDB 3PM0. b, Dose responses in CYP1B1 activity assay. c, Compari-son of selectivity and potency for 1-3. 
A)

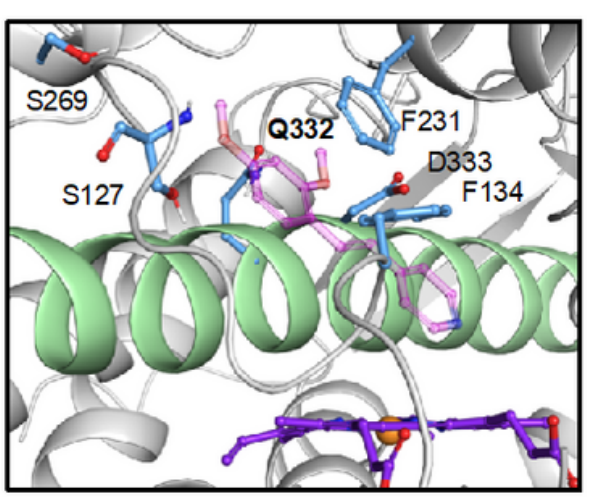

B)

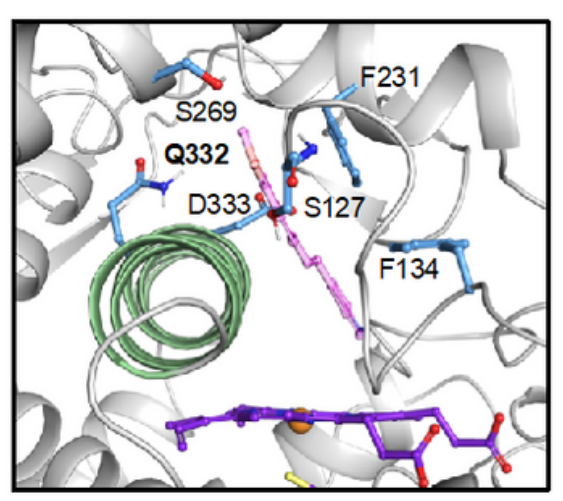

C)

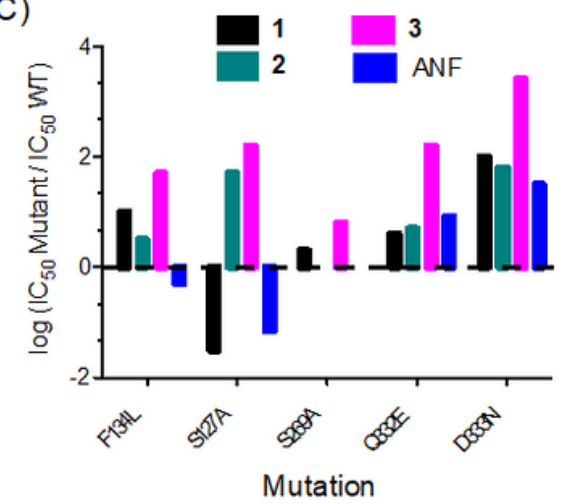

Figure 2

Mutations reveal key contacts in CYP1B1. a, b, Location of mutated amino acids. The l-helix is shown in green, and the heme in purple. Q332 (bold) is not found in other CYP1 family members. F231 is essential for turnover of the REE substrate. c, Effect of point mutations on the efficacy of CYP1B1 inhibitors. The inhibitors were less potent in the mu-tants, with the exception of ANF and 1 in the S127A mutant and ANF in the F134L mutant. The log of the ratio of IC50 values was used to simplify visualization. See Table S2 for all values.

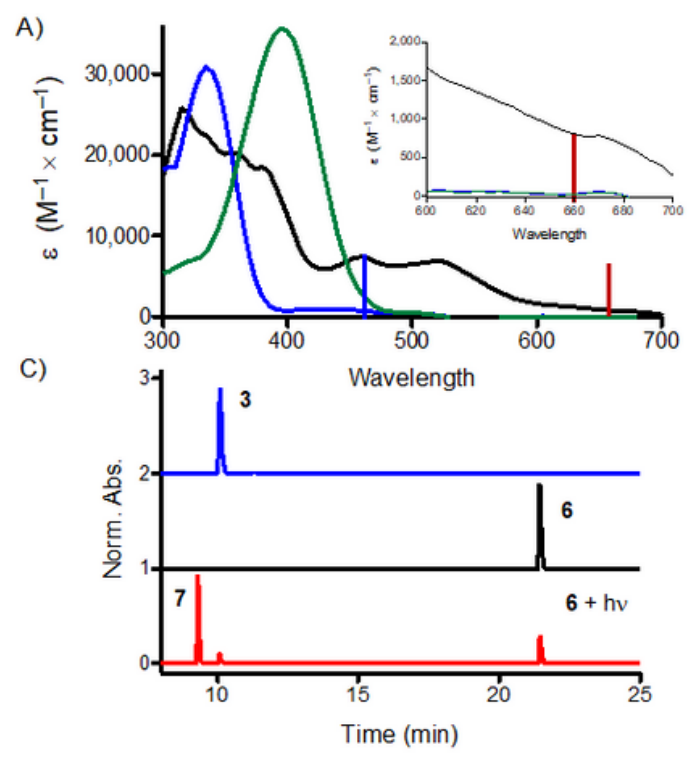

B)
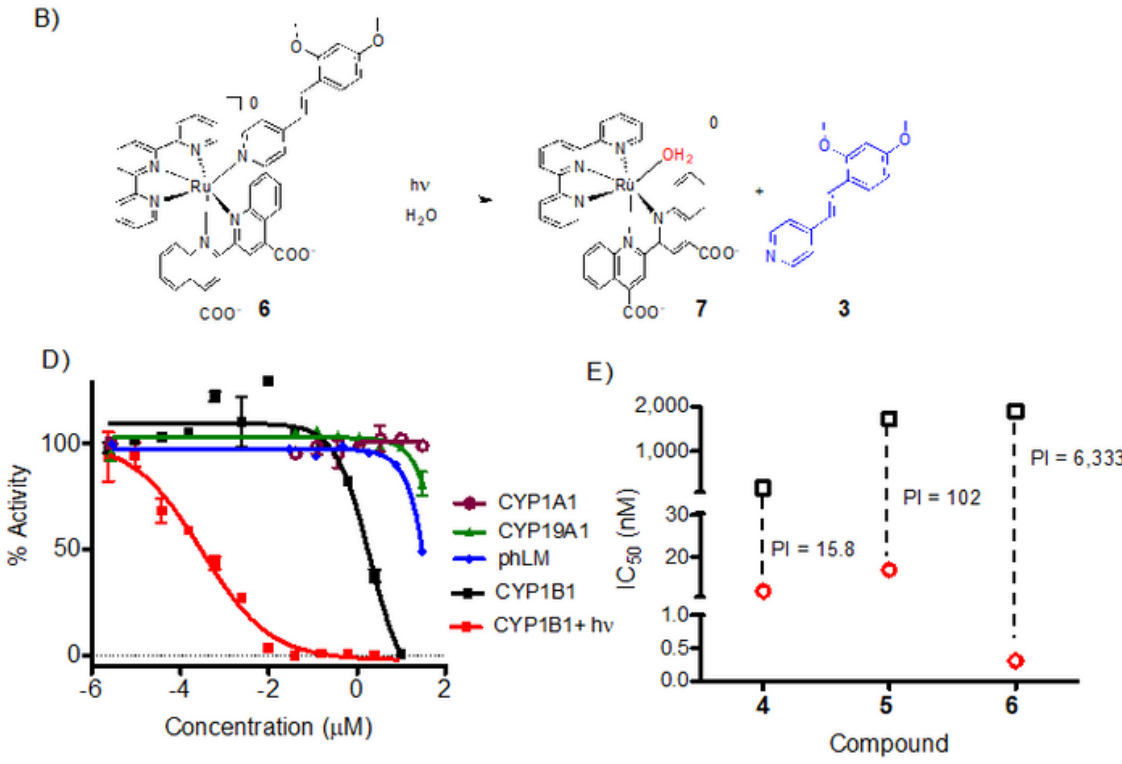

\section{Figure 3}

Photocontrol of a Ru(II) complex containing a CYP1B1 inhibitor. a, Absorption spectra in $\mathrm{CH} 3 \mathrm{CN}$ of 6 (black), 3 (blue), and 3 following protonation with $5 \mathrm{mM} \mathrm{HCl}$ (green). The excitation wavelengths for the blue and red LEDs are indicated with vertical bars. Inset shows an expansion of the 600-700 nm region. b, Photoejection scheme for 6 in water. c, HPLC analysis showing the chromatograms for 3, 6, and 6 following irradiation. The formation of complex 7 and in-hibitor 3 were verified by UV/Vis. The protonated 
form of 3 was observed. d, Dose responses for 6 in the dark with CYP1A1, 19A1, and pooled human liver microsomes, CYP1B1, and with 1B1 following irradiation $(58.7 \mathrm{~J} / \mathrm{cm} 2)$. e, Com-parison of potency and PI values for light activated $\mathrm{Ru}(\mathrm{II})$ complexes 4-6 demonstrate a rage of 103 due to improvement in both the inhibitor and $\mathrm{Ru}(\mathrm{II})$ scaffold.

\section{Supplementary Files}

This is a list of supplementary files associated with this preprint. Click to download.

- PotentandSelectiveSI092021.docx

- Chart.png 